\title{
Pre-0perative Hyponatremia in a High Dependency Cardiovascular Surgical Center of a Tertiary Health Facility in Nigeria: Risk Factors, Prevalence, Clinical Correlates and Treatment Outcome. A Retrospective Cohort Study
}

\author{
Peter K. Uduagbamen ${ }^{1 *}$ (1), Michael Sanusi², Olumide B. Udom², Omotayo F. Salami', \\ Adedeji D. Adebajo², Oluwaseyi J. Alao1, Osaze Ehioghae1 ${ }^{1}$ \\ ${ }^{1}$ Division of Nephrology and Hypertension, Department of Internal Medicine, Ben Carson (Snr) School of Medicine, Babcock \\ University/Babcock University Teaching Hospital, Ilishan-Remo, Nigeria \\ ${ }^{2}$ Tristate Heart and Vascular Centre, Babcock University Teaching Hospital, Ilishan-Remo, Nigeria \\ ${ }^{3}$ Intensive Care Unit, Department of Surgery, Ben Carson (Snr) School of Medicine, Babcock University/Babcock University \\ Teaching Hospital, Ilishan-Remo, Nigeria \\ Email: ${ }^{*}$ petr.uduagbamen@gmail.com,michaelosanusi@gmail.com, bazudom@yahoo.co.uk, tsalami40@gmail.com, \\ jradebajo@yahoo.co.uk, oluwaseyialao26@gmail.com,dr_ziggszz@yahoo.com
}

How to cite this paper: Uduagbamen, P.K., Sanusi, M., Udom, O.B., Salami, O.F., Adebajo, A.D., Alao, O.J. and Ehioghae, O. (2020) Pre-Operative Hyponatremia in a High Dependency Cardiovascular Surgical Center of a Tertiary Health Facility in Nigeria: Risk Factors, Prevalence, Clinical Correlates and Treatment Outcome. A Retrospective Cohort Study. Open Journal of Internal Medicine, 10, 288-301.

https://doi.org/10.4236/ojim.2020.103030

Received: August 19, 2020

Accepted: September 26, 2020

Published: September 29, 2020

\begin{abstract}
Introduction: Hyponatremia is the commonest electrolyte derangement seen in medical and surgical wards and in high dependency treatment units. It is associated with prolonged hospital stay, high economic burden and increased morbidity and mortality. Hyponatremia is well reported in medical and surgical conditions but literature is scanty concerning its relationship with treatment outcome in patients managed in high dependency heart and vascular surgical centers. Objectives: To determine the prevalence, determinants, clinical correlates and outcome of pre-operative hyponatremia. Methods: Retrospective study involving patients that had surgery (cardiac and vascular) between January 2015 and December 2019. Data were obtained from patients' case files, perioperative and intensive unit charts. All participants with preoperative hyponatremia had their serum sodium corrected to $\geq 135 \mathrm{mmol}$ before surgical incision and those with hypernatremia had their sodium corrected to $<145 \mathrm{mmol}$. The socio-demographic, clinical, laboratory findings were entered. Statistical analysis was done using student's t-test and Chi square. Results: Of the 305 participants (186 males and 119 females), pre-operative
\end{abstract}


Copyright $\odot 2020$ by author(s) and Scientific Research Publishing Inc. This work is licensed under the Creative Commons Attribution International License (CC BY 4.0).

http://creativecommons.org/licenses/by/4.0/ (c) (i) Open Access hyponatremia was found in $30.8 \%$ and normonatremia in $59.7 \%$. Pre-operative hyponatremia was more common in advance age, overweight, those presenting for open heart and other major surgeries and those with background chronic heart failure and chronic liver disease. Participants with pre-operative hyponatremia had shortened and also prolonged hospital stay and higher risk of death than with normonatremia. Conclusion: Pre-operative hyponatremia is common in high dependency surgical unit particularly in the elderly, those with comorbidities and those presenting for major heart surgeries. It impacts negatively on the morbidity and mortality as it could shorten hospital stay through death, and could prolong hospital stay with increased health burden on patients, their care givers and the general population. The impact of this finding across different races needs further evaluation.

\section{Keywords}

Hyponatremia, Normonatremia, Comorbidities, Pre-Operative, Sodium

\section{Introduction}

Sodium is the most abundant electrolyte in the extracellular fluid compartment and with this, it determines the osmolality and tonicity of the blood. However, the osmolality and tonicity of the intracellular space is determined and related to water balance [1]. Due to its close association with water, it mediates the interplay of fluid exchanges, volume and pressure balances in the extracellular and intracellular spaces through various transport pathways [2]. Because of its involvement in multiple cellular activities, derangement in sodium levels often lead to disturbances of functions in many organ systems in the body and these could present as dehydration, edema or dilution of plasma. Hyponatremia is common in surgical intensive care units and contributes significantly to the morbidity and mortality in these patients from derangements in cellular activities, particularly of the brain, heart, lungs, liver and kidneys [3]. Pre-operative hyponatremia has been attributed to various factors like diuretic use, renal tubular acidosis, advance age and poor salt diet often needed to control hypertension, chronic kidney disease (CKD), liver disease and heart failure [4]. Hyponatremia is also a common finding in marathoners, worse still, under heat and dehydration leading to thirst, and vasopressin release [5]. Arterial blood under filling with relative or absolute tissue hypoperfusion causes vasopressor release leading to a state of effective hypovolemia associated with hypotonicity. There are various mechanisms leading to hyponatremia in health and in disease. Overall, these mechanisms are mediated either through osmotic vasopressor stimulation, by thirst (or salt appetite) or though the sympathetic nervous system (SNS), which is not osmotically mediated [6]. Hyponatremia found in heart failure, cirrhosis, chronic pain, cancer and stroke are commonly secondary to stimulation, at times, excessive, of the antidiuretic hormone $(\mathrm{ADH})$ or from dilution of 
plasma from fluid overload (hypovolemic hyponatremia) [7]. Tannor et al. found infection as the commonest associate of hyponatremia on the medical wards in Ghana [8]. When it arises from diuretic use, it is associated with tubular dysfunction with poor sodium adsorption with or without potassium secretion [9]. Whatever the mechanism is, once developed, hyponatremia is commonly symptomatic, though less in uremic states, due to higher blood urea which acts as an effective osmolyte [10]. Symptoms are commonly dictated by the duration and rate of fall of serum sodium. Acute hyponatremia, developing within 48 hours, often manifest dysfunction of the nervous system ranging from mild cognitive decline to convulsion, motor paralysis and death [11]. Chronic disease is usually less debilitating as the nervous system could mount counter regulating mechanisms that involves the recruitment of organic osmolytes which regulate the rate of fluid diversion away from neurons to the glial tissues thereby preventing cerebral edema. When these compensatory mechanisms are overwhelmed, especially with rapid fall in plasma sodium, it could lead to the development of osmotic demyelination injury to the cells of the central nervous system particularly the pons, the cerebellum, lateral geniculate body (LGB), internal capsule, hippocampus and structures of the basal ganglia. Over correction or too rapid rise in serum levels can also result in osmotic demyelinating injury [12].

Many authors have reported hyponatremia in intensive care units mostly related to medical conditions but much is still unknown concerning hyponatremia related to surgeries particularly cardiovascular surgeries, its causes, correlates and outcome. We studied pre-operative hyponatremia in order to determine its risk factors and prevalence, clinical correlates and treatment outcome in patients presenting for cardiac and vascular surgeries in a high dependency heart and vascular surgical center of a teaching hospital.

\section{Patients and Methods}

This was a retrospective study carried in a high dependency cardiac and vascular surgical unit in a tertiary health institution in Ilishan-Remo. Tristate Heart and Vascular Center is a higher dependency center which is dedicated to heart and vascular repair, replacement and implantation procedures in Southwest Nigeria, The hospital receives patients across all ages from all parts of Nigeria and the West Africa sub-region. Case notes and perioperative charts of patients treated in the center from January 2015 to December 2019 were retrieved. Variables obtained from the case files included age, gender, type and cause of cardiovascular disease, duration of disease, previous surgical procedure related to disease, class of surgery, body mass index (BMI), percentage oxygen saturation, pulse rate, blood pressures (BP), heart rate, duration of hospital stay till discharge or death and comorbidities (heart failure, chronic liver disease, chronic kidney disease, stroke or cancer). Patients less than 16 years were excluded from the study. The serum electrolytes, albumin, packed cell volume (PCV) and blood glucose at admission and at post-operative day (POD) 28 were documented. All partici- 
pants with pre-operative hyponatremia had their serum sodium corrected with Oral Sodium bicarbonate $300 \mathrm{mg}$ thrice daily until sodium $>135.0 \mathrm{mmol}$ was achieved. At the same time, participants with serum potassium $<3.8 \mathrm{mmol}$ were also place on Oral Potassium chloride $600 \mathrm{mg}$ twice daily to counter the potassium-lowering feature of sodium bicarbonate therapy.Participants with hypernatremia had their serum sodium corrected. For participants who were discharged before their POD 28, they were reviewed at 4 weeks post-surgery according to unit protocols. Where infection occurred, type and site were documented. Infusions and inotropes used were documented. Admissions less than 48 hours was excluded.

Definitions:

Hyponatremia, serum sodium $<135.0 \mathrm{mmol}$ [8]

Normonatremia, serum sodium 135.0 - $145.0 \mathrm{mmol}$ [9]

Hypernatremia, serum sodium $>145.0 \mathrm{mmol}$ [12]

Data obtained were analyzed using IBM SPSS software version 22 (IBM Inc, NY, USA). Data were presented in tables. Continuous variables were expressed as means and standard deviations and compared using student t-test. Categorical variables were expressed as frequencies and percentages and compared using Chi square test. $\mathrm{P}<0.05$ was considered statistically significant.

\section{Results}

Four hundred and ninety nine patients had surgeries in the center. Out of this, 148 were excluded on account of being less than 16 years, 32 for incomplete data and 14 on account of previous surgeries within 30 days to the index surgery. Of the 305 that met the inclusion criteria and participated in the study, 102 had open heart surgery, 90 had major vascular surgery, 15 had intermediate grade surgery and 98 had minor surgeries. One hundred and eighty six (61.0) were males and 119 (39.0) were females. The mean age of the participants was $52.8 \pm$ 143 years, with males $51.3 \pm 6.6$ and females, $54.1 \pm 3.7$. The demographic and clinical characteristics of the participants is shown in Table 1. A greater proportion of participants, 126 (41.3), were between 40 and 59 years followed by the elderly 91 (29.8). The commonest presenting compliant was difficulty with breathing 107 (35.1) followed by chest pain 93 (30.5). A greater proportion of participants were overweight 91 (29.9) followed by those with normal BMI, 88 (28.9). Sixty nine (22.6) participants had comorbidities, 56 (81.1) of these were males with 13 (18.9) females. Ninety seven percent of participants with comorbidities had hyponatremia. Majority 82 (87.2) of participants with pre-op hyponatremia were on antihypertensive drugs and of this, 67 (81.7) were receiving diuretic for blood pressure control.

The laboratory parameters of the participants are showed in Table 2. The mean sodium at admission was significantly higher than at POD 28, 132. $3 \pm 3.5$ and 127.5.6 respectively, $\mathrm{P}=0.03$. The mean sodium of the male and female participants at admission were $131.6 \pm 8.2$ and $134.9 \pm 11.2 \mathrm{mmol} / \mathrm{l}(\mathrm{P}=0.04)$, and 
Table 1. Socio-demographic characteristics of the participants.

\begin{tabular}{clcc}
\hline Variables & & Frequency & Percentage \\
\hline Genders & Females & 119 & 39.0 \\
& Males & 186 & 61.0 \\
Age, years & $16-39$ & 88 & 28.9 \\
& $40-59$ & 126 & 41.3 \\
& $\geq 60$ & 91 & 29.8 \\
BMI, kg/m ${ }^{2}$ & $<19.5$ & 71 & 23.3 \\
& $19.5-24.9$ & 88 & 28.9 \\
& $25.0-29.9$ & 91 & 29.8 \\
Surgery type & $>30.0$ & 55 & 18 \\
Cardiac surgery & & 102 & 33.5 \\
Major Vascular & & 90 & 29.5 \\
Intermediate & & 15 & 4.9 \\
Minor & & 98 & 32.1 \\
\hline
\end{tabular}

BMI-body mass index.

Table 2. Laboratory results of the participants.

\begin{tabular}{ccccc}
\hline Variables & Pre-op N = 305(\%) & 4 weeks N = 305 (\%) & $\mathrm{X}^{2}$ & P-value \\
\hline Sodium, mmol/1 & & & & \\
$\leq 125$ & $7(2.3)$ & $19(6.2)$ & 5.3 & 0.001 \\
$125-134.9$ & $87(28.5)$ & $163(53.5)$ & & \\
$135-145$ & $211(69.2)$ & $123(40.3)$ & & \\
\hline
\end{tabular}

at POD 28 were $125.8 \pm 4.6$ and $129.7 \mathrm{mmol} / \mathrm{l}(\mathrm{P}=0.03)$ respectively.

A greater proportion of participants 211 (69.2) had normal serum sodium at admission but post-operatively, a greater proportion had hyponatremia 182 (59.7). Participants $\geq 60$ years had the greater proportions of participants with hyponatremia at both admission and at POD 28, 61 (64.9) and 83 (45.6) respectively. The clinical and laboratory correlates of hyponatremia are shown in $\mathrm{Ta}$ ble 3. The percentage of females that had hyponatremia was less than those that had normonatremia while the percentage of males with hyponatremia was less than those with normonatremia, $P=0.03$. A greater percentage of participants with hyponatremia at admission and at POD 28 had cardiac surgery or a major vascular surgery compared to those that had minor and intermediate grade surgeries, $\mathrm{P}<0.001$. As the duration of hospital stay increased, frequency of hyponatremia increased. The percentage of participants with pre-op hyponatremia was less compared with the percentage of hyponatremic participants at POD 14 and beyond, (30.8\% versus $49.3 \%), \mathrm{P}<0.001$. Forty five (14.8) participants had infections on admission, of these, 32 (71.1) were localized, while 13 (28.9) were 
Table 3. Demographic, clinical and laboratory correlates of hyponatremia

\begin{tabular}{|c|c|c|c|c|c|}
\hline Variables & $\begin{array}{c}\text { Frequency } \\
\mathrm{N}=305(\%)\end{array}$ & $\begin{array}{c}\text { Pre-op } \mathrm{Na}<135 \\
\mathrm{~N}=94(\%) \\
\text { Mean } \pm \mathrm{SD}\end{array}$ & $\begin{array}{c}\text { Pre-op Na } 135-145 \\
\text { N = 211 (\%) } \\
\text { Mean } \pm \text { SD }\end{array}$ & $\mathrm{X}^{2}$ t-test & P-value \\
\hline \multicolumn{6}{|l|}{ Gender } \\
\hline Females & $119(39.0)$ & $32(34.0)$ & $87(41.2)$ & 2.6 & 0.02 \\
\hline Males & $186(61.0)$ & $62(66.0)$ & $124(58.8)$ & & \\
\hline \multicolumn{6}{|l|}{ Age, years } \\
\hline $16-39$ & $88(28.9)$ & $8(8.5)$ & $80(37.9)$ & 4.4 & 0.001 \\
\hline $40-59$ & $126(41.3)$ & $25(26.6)$ & $101(47.9)$ & & \\
\hline$\geq 60$ & $91(29.8)$ & $61(64.9)$ & $30(14.2)$ & & \\
\hline \multicolumn{6}{|l|}{ Surgeries } \\
\hline Cardiac & $102(33.5)$ & $51(54.3)$ & $51(24.2)$ & 5.6 & $<0.001$ \\
\hline Major Vascular & $90(29.5)$ & $27(28.7)$ & $63(29.9)$ & & \\
\hline Intermediate & $15(4.9)$ & $5(5.3)$ & $10(4.7)$ & & \\
\hline Minor & $98(32.1)$ & $11(11.7)$ & $87(41.2)$ & & \\
\hline \multicolumn{6}{|l|}{ BMI, $\mathrm{kg} / \mathrm{m}^{2}$} \\
\hline$<19.5$ & $71(23.3)$ & $9(9.6)$ & $62(29.4)$ & 2.9 & 0.002 \\
\hline $19.5-24.9$ & $88(28.9)$ & $17(18.1)$ & $71(33.6)$ & & \\
\hline $25.0-29.9$ & $91(29.8)$ & $46(48.9)$ & $45(21.3)$ & & \\
\hline$>30.0$ & $55(18.0)$ & $22(23.4)$ & $33(15.7)$ & & \\
\hline Mean SBP, mmHg & $305(100)$ & $152.1 \pm 9.2$ & $132.5 \pm 4.6$ & 6.3 & $<0.001$ \\
\hline Mean DBP, mmHg & $305(100)$ & $95.4 \pm 7.2$ & $83.6 \pm 2.3$ & 4.3 & $<0.001$ \\
\hline Mean $\mathrm{SPO}_{2},(\%)$ & $305(100)$ & $95.2 \pm 8.8$ & $97.7 \pm 9.5$ & 6.4 & $<0.001$ \\
\hline \multicolumn{6}{|l|}{ Serum biochemistry } \\
\hline Hematocrit, \% & $305(100)$ & $33.6 \pm 3.8$ & $38.4 \pm 8.1$ & 5.9 & $<0.001$ \\
\hline Albumin, mg/dl & $305(100)$ & $35.1 \pm 11.5$ & $33.9 \pm 9.8$ & 0.9 & 0.07 \\
\hline Creatinine, umol/l & $305(100)$ & $103.4 \pm 14.2$ & $111.7 \pm 5.6$ & 5.1 & $<0.001$ \\
\hline eGFR, $\mathrm{ml} / \mathrm{min}$ & $305(100)$ & $68.3 \pm 5.2$ & $60.8 \pm 9.7$ & 6.8 & $<0.001$ \\
\hline Urea mmol/l & $305(100)$ & $46.7 \pm 3.8$ & $71.2 \pm 7.8$ & 6.2 & $<0.001$ \\
\hline Chloride, $\mathrm{mmol} / \mathrm{l}$ & $305(100)$ & $100.5 \pm 6.9$ & $96.1 \pm 4.2$ & 5.4 & $<0.001$ \\
\hline Bicarbonate, $\mathrm{mmol} / \mathrm{l}$ & $305(100)$ & $23.8 \pm 4.6$ & $20.1 \pm 7.2$ & 4.9 & $<0.001$ \\
\hline Potassium, mmol/l & $305(100)$ & $4.7 \pm 2.2$ & $4.6 \pm 3.4$ & 0.7 & 0.8 \\
\hline \multicolumn{6}{|l|}{ Days on admission } \\
\hline$<7$ & $143(46.9)$ & $19(20.2)$ & $124(58.8)$ & 6.3 & $<0.001$ \\
\hline $7-13$ & $22(7.2)$ & $6(6.4)$ & $16(7.6)$ & & \\
\hline $14-27$ & $106(37.8)$ & $47(50.0)$ & $59(27.9)$ & & \\
\hline$>28$ & $34(1.1)$ & $22(23.4)$ & $12(5.7)$ & & \\
\hline Comorbidities & & & & & \\
\hline
\end{tabular}


Continued

\begin{tabular}{ccccc}
\hline None & $236(77.4)$ & $49(52.1)$ & $187(88.6)$ & $<0.001^{*}$ \\
1 & $44(14.4)$ & $25(26.6)$ & $19(9.0)$ & \\
2 & $18(5.9)$ & $13(13.8)$ & $5(2.4)$ & \\
3 & $7(2.3)$ & & $7(7.5)$ & $0(0.0)$
\end{tabular}

POD—post operative day, Na-sodium, SD—standard deviation, BMI-body mass index, SBP—systolic blood pressure, $\mathrm{DBP}$ - diastolic blood pressure, $\mathrm{SPO}_{2}$ - percentage oxygen saturation, ${ }^{*}$ - fisher's exact test.

Table 4. Multivariate regression analysis.

\begin{tabular}{cccc}
\hline Variables & OR & $95 \% \mathrm{CI}$ & P-value \\
\hline Sex & 1.32 & $1.26-1.46$ & 0.06 \\
Age & 7.82 & $5.63-8.47$ & 0.02 \\
Surgery type & 0.048 & $0.012-0.153$ & $<0.001$ \\
Comobidities & 2.62 & $1.44-7.67$ & $<0.001$ \\
Length of admission & 1.23 & $1.14-2.91$ & 0.001 \\
BMI & 5.56 & $5.27-7.11$ & 0.04 \\
SBP & 9.63 & $7.88-11.34$ & 0.002 \\
DBP & 2.92 & $2.74-2.99$ & 0.05 \\
SPO 2 & 0.03 & $0.02-0.85$ & $<0.001$ \\
eGFR & 4.35 & $2.74-7.55$ & 0.001 \\
Biocarbonate & 0.23 & $0.21-1.46$ & $<0.001$ \\
\hline
\end{tabular}

OR—odd ratio, $\mathrm{CI}$ - confidence interval, BMI—body mass index, SBP—systolic blood pressure, DBP—diastolic blood pressure, $\mathrm{SPO}_{2}$ - percentage oxygen saturation, eGFR-estimated glomerular filtration rate.

sepsis. All participants with sepsis had hyponatremia. Six (46.2) of the septic patients died out the 9 (3.0) deaths recorded within study duration. Of the dead, 2 (22.2) had hyponatremia on admission and 5 (55.6) at POD 28. Two (22.2) of the dead died within the POD 3 while 7 (77.7) stayed beyond POD 28 on admission. Comorbidities were more in males as they made up $66.0 \%$ of participants with pre-op hypoatremia but $69.6 \%$ of participants with comorbidities, $\mathrm{P}=0.003$

Multivariate regression analysis in Table 4 showed age (OR-7.82, CI-5.63 8.47), length of hospital stay (OR-1.23 CI-1.14 - 2.91), BMI (OR-5.56 CI5.27 - 7.11), SBP (OR-9.63 CI-7.88 - 11.34), $\mathrm{SPO}_{2}(\mathrm{OR}-0.03$, $\mathrm{CI}-0.02-0.85)$, eGFR (OR-4.35, CI-2.74 - 7.55) and Bicarbonate (OR-0.23, CI-0.21 - 1.46) were predictors of pre-operative hyponatremia.

\section{Discussion}

The prevalence of pre-operative hyponatremia in this study was $30.8 \%$ and this rose to $59.7 \%$ at POD 28. The prevalence of pre-operative normonatremia was $69.2 \%$. This prevalence of hyponatremia is within the range reported by Madhuvan et al. in a review and gave prevalences ranging from $3.4 \%$ to $39.4 \%$ [13]. It is however higher than that found by Cecconium et al. who reported $23.8 \%$ [14]. 
This difference can be attributable to differences in the cut-off for serum sodium as Cecconium used $<137 \mathrm{mmol} / \mathrm{l}$ as against $<135 \mathrm{mmol} / \mathrm{l}$ used in this study. Hyponatremia became even more prevalent as these patients had surgery and went on admission. This pattern agrees with findings by Leung et al. who reported that pre-op hyponatremia was a risk factor for perioperative morbidity and mortality and further worsening of the hyponatremia [15]. Hyponatremia was commoner and more severe in males in this study. This finding is in disagreement with a previous study that found hyponatremia commoner in females [16]. It is however in agreement with Mannheimer et al. who reported that hyponatremia and comorbidities associated with it are commoner in men [17]. Moreover, in this study, apart from comorbidities being commoner in males, these males were more likely to develop hyponatremia compared with females. The common comorbidities found in participants in this study were heart failure and chronic diseases of the kidneys and the liver, conditions associated with upregulation of the vasopressor mechanisms of the body mediating secretion of the antidiuretic hormone (ADH) that produce salt poor water retention [18]. Females tend to be more affected in hyponatremia from the addictive effects of estrogens and vasopressin on the $\mathrm{Na}^{+} \mathrm{K}^{+}$ATPase on the central nervous system [19].

Hyponatremia was found more in the elderly than in the younger groups in this study. Kirby et al. who reported that advanced age was a risk factor for developing hyponatremia [20]. Miller et al. also found an inverse relationship between serum sodium and age, they reported age related prevalence of hyponatremia as $7.7 \%$ at less than 55 years, and $11.6 \%$ at greater than 75 years [21]. The higher prevalence of hyponatremia in the elderly population could be secondary to reduced water excretion from declining glomerular filtration (GFR), reduced production of prostaglandins (PGs), increased sensitivity to osmotic stimulation especially through thirst, reduction in total body water (TBW), high prevalence of usage of sodium losing dugs like antidepressants and the frequent restrictions on their salt intake, as part of blood pressure control [22]. The higher incidence of hyponatremia in participants presenting for major surgeries particularly cardiac surgery was also reported by Saepudin et al. [23] who found more incidences of hyponatremia in individuals with chronic heart, liver and kidney disease. The reduced cardiac reserve coupled with systolic dysfunction reduce the ejection fraction and the effective cardiac output leading to relative organ perfusion which mediate the release of vasopressors with sodium poor fluid reduction [24]. Hyponatremia was commoner as the BMI increased in the study, this agrees with Pandey et al. who found a significant negative correlation between serum sodium and the BMI [25]. Our finding is however in disagreement with Qureshi et al. who found a positive correlation sodium and BMI [26]. Higher weights are associated with an increase in the central pool causing higher cardiac output and stroke volume. The association between hyponatremia and low bone density with cardiac fibrosis and calcific depositions in the heart lead to poor systolic and diastolic function [27]. This has a synergistic effect with the poor 
cardiac reserve and contributes to the relatively lower peripheral resistance in the obese secondary to suppressed sympathetic output. This, coupled with the relative hemodilution seen in the obese, results in hyponatremia [28].

The association between hypertension and hyponatremia in this study agrees with findings by Israel et al. who reported higher incidences of hyponatremia in subjects with elevated high density cholesterol (HDL) [29]. Though lipids were not assayed for in this study, hyponatremia in hypertensives could be multifactorial. The use of thiazides and even loop diuretics could lead to chronic hyponatremia [30]. The tubular dysfunction induced by diuretics particularly the thiazides and the thiazide like drugs suppress sodium reabsorption thereby leading to increased secretion into the urine. Again, the low sodium diet often recommended for this group also contribute to the hyponatremia [31]. Metabolic acidosis was associated with hyponatremia in this study. Sahay et al. reported hyponatremia associated with renal tubular acidosis (RTA) and this is commonly secondary to tubular dysfunction which can be induced by some drugs, in addition to the ones listed above, used in these patients (to treat hypertension, heart failure and chronic liver disease particularly portal hypertension) particularly aldosterone antagonist altering the activities of the principal cells of the distal tubules [32]. Serum albumin was not significantly related to serum sodium level in this study. Jhaveri et al. had earlier reported a positive relationships between blood levels of albumin and sodium [33]. Hyponatremia and hypoalbuminemia commonly occur together in and are poor prognostic factors in chronic liver disease and heart failure. The lack of significant association in our study, unlike in the above studies, could be due to the fact that most of the participants in this study were more healthier, more likely to have better appetite, lesser inflammation and lower risk of protein energy malnutrition (PEM) compared to the studies above, whose subjects had worse clinical conditions. Anemia was a risk factor for developing hyponatremia in this study similar to findings by Yamada et al. [34] Anemia and hyponatremia are common findings in chronic medical conditions. The occurrence of anemia, outside blood loss, could be of hypoproliferative origin and this can be secondary to kidney disease, chronic anorexia, malnutrition and PEM. Tseng et al. reported higher prevalence of hyponatremia, (in association with hypouricemia) associated with anemia in the elderly population [35]. In this study, hyponatremia was more prevalent in participants with background CKD and this agrees with authors that reported various mechanisms through which the relationship between elevated nitrogenous waste (from declining glomerular filtration) and hyponatremia are mediated. Low level sodium, but more importantly, chloride is needed to stimulate renin secretion and for the activation of the tubuloglomerular feedback mechanism [36]. The finding of low chloride with high renin levels, for instance in Barters' syndrome, underlines this interplay. This also explains the lower efficacy of diuretics and inhibitors of the renin angiotensin aldosterone system (RAAS) in the presence of hyponatremia but even more so with hypochloremia [37]. The tubular defects induced by low- 
er sodium and chloride also play a part in the alteration of the balance between secretory activity of the basolateral membranes and absorption by the apical membranes and this could cause disease activity in CKD out of proportion to serum creatinine, mimicking states of PEM and dialysis cachexia [38]. However, its worth noting that secretory and absorptive functions of the tubules are also dependent on the acid-base balance hence the function of maintaining the acid base balance in the tubular $\alpha$-intercalated could be compromised in states of excessive reductions in serum chloride and sodium even in the presence of augmented absorptive functions of the principal cells of the distal tubules [39].

There was an inverse relationship between the serum sodium and the length of hospital stay in this study. This is in agreement with findings by Berandil et al. who found that hyponatremia was a risk factor for prolonged hospital stay [40]. We observed that those that stayed in less than 7 days had the lowest prevalence of hyponatremia compared to those that stayed beyond POD 28. There was a positive correlation between the duration of hospital stay and mortality and this is similar to findings by et al. [41]. Hyponatremia is an oxidative stress or state and disrupt the oxidation-reduction potential of cells leading to dysfunctional transport pathways particularly the cellular membrane energy requiring glucose phosphorylation needed for building up the energy stores of the body.

The management of hyponatremia is guided by serum levels, duration of disease, rate of sodium fall and disease symptomatology [42]. Preventive measures aimed at reducing the incidences of hyponatremia could be slowed down by the age long advice to hypertensive and CKD patients to reduce salt intake. Diuretics therapy usually need review and possible replacement especially in the preparation for elective surgery. In acute cases, $3 \%$ saline can be used with loop diuretics and fluid restriction to $<1$ Litre/day with a target of sodium rise not exceeding 10 - $12 \mathrm{mmol} / \mathrm{l}$ in $24 \mathrm{hrs}$ or a $1 \mathrm{mmol} / \mathrm{l}$ per hour increase [43]. In chronic cases, suppression of vasopressors activities is often needed with V2 antagonist Vaptans [43]. Overzealous corrections are always avoided to prevent osmotic demyelination syndrome, and when this occur, management involves gradual reductions in serum sodium.

Some limitations encountered in this study included the fact that many of the participants that had minor and intermediate grade surgeries had no arterial blood gases result nor central venous pressure recordings hence acid-base balance and volume status could not be deeply assessed beyond that provided by bicarbonate and vital signs respectively. Data on comorbidities were retrieved from patient case notes, history and examination, so it is possible there could be some comorbid conditions that were not discovered. Data on inotropic support were unavailable for some of participants with minor and intermediate surgeries.

\section{Conclusion}

Pre-operative hyponatremia is a common finding among surgical patients presenting for surgery, more so as they come into admission, with a prevalence of 
$30.8 \%$. Risk factors identified for this included male gender, advance age, diuretic use, high BMI, elevated blood pressure, metabolic acidosis, anemia, and comorbidities like cardiac, hepatic, renal diseases. Participants with pre-op hyponatremia had higher risk for prolong hospital, severe hyponatremia, poor response to treatment and death. It is therefore important comorbid conditions are properly screened for and manage before elective cardiac and vascular surgeries to reduce the health burden, morbidity and mortality from these procedures. Our study was a single centered, therefore more studies, particularly multicenter and population based, involving all race and ages, are needed for the formulation of a comprehensive management approach.

\section{Acknowledgements}

The nursing and the technical staff of Tristate Heart and Vascular Centre, for their assistance in retrieving the case notes and the peri and post-operative charts for data collection.

\section{Conflicts of Interest}

The authors declare no conflicts of interest regarding the publication of this paper.

\section{References}

[1] Sterns, R.H. (2015) Disorders of Plasma Sodium-Causes, Consequences, and Correction. The New England Journal of Medicine, 372, 55-65. https://doi.org/10.1056/NEJMra1404489

[2] Roumelioti, M.E., Glew, R.H., Khitan, Z.J., Berrios, H.R., Argyropoulos, C.P., Malhotra, D., et al. (2018) Fluid Balance Concepts in Medicine: Principles and Practice. World Journal of Nephrology, 7, 1-28.

[3] El-Sharkawy, A.M., Sahota, O., Maughan, R.J. and Lobo, D.N. (2014) The Pathophysiology of Fluid and Electrolyte Balance in the Older Adult Surgical Patients. Clinical Nutrition, 33, 6-13. https://doi.org/10.1016/j.clnu.2013.11.010

[4] Bhave, G. and Neilson, E.G. (2011) Volume Depletion versus Dehydration: How Understanding the Difference Can Guide Therapy. American Journal of Kidney Diseases, 58, 302-309. https://doi.org/10.1053/j.ajkd.2011.02.395

[5] Knechtle, B., Chlibkoya, D., Papadopoulou S, Mantzorou M, Rosemann T, Nikolaidis $\mathrm{P}$ et al. (2019) Exercise-Associated Hyponatremia in Endurance and Ultra-Endurance Performance-Aspects of Sex, Race Location, Ambient Temperature, Sports Discipline, and Length of Performance: A Narrative Review. Medicina, 55, 537. https://doi.org/10.3390/medicina55090537

[6] Martin, J., Burnier, M. and Lu, H.R. (2018) [Approach to the Syndrome of Inappropriate Antidiuretic Hormone Secretion (SIADH)]. Revue Médicale Suisse, 14, 2116-2120.

[7] Böhm, M., Drexler, H., Oswald, H., Rybak, K., Bosch, R., Butter, C., Klein, G., Gerritse, B., Monteiro, J., Israel, C., et al. (2016) Fluid Status Telemedicine Alerts for Heart Failure: A Randomized Controlled Trial. European Heart Journal, 37, 3154 3163. https://doi.org/10.1093/eurheartj/ehw099

[8] Tannor, E.K., Akumiah, E.O. and Norman, B.R. (2019) Outcomes in Medical Ad- 
missions with Hyponatremia in Ghana-A Single-Centre Study. African Journal of Nephrology, 22, 40-45. https://doi.org/10.21804/22-1-3306

[9] Fortes, M.B., Owen, J.A., Raymond-Barker, P., Bishop, C., Elghenzai, S., Oliver, S.J. and Walsh, N.P. (2015) Is This Elderly Patient Dehydrated? Diagnostic Accuracy of Hydration Assessment Using Physical Signs, Urine, and Saliva Markers. Journal of the American Medical Directors Association, 16, 221-228. https://doi.org/10.1016/j.jamda.2014.09.012

[10] Kubota, K., Sakaguchi, Y., Hamano, T., Oka, T., Yamaguchi, S., Shimada, K., et al. (2018) Prognostic Value of Hypochloremia versus Hyponatremia among Patients with Chronic Kidney Disease-A Retrospective Cohort Study. Nephrology Dialysis Transplantation, 35, 987-994. https://doi.org/10.1093/ndt/gfy299

[11] Rondon-Berrios, H., Agaba, E.I. and Tzamaloukas, A.H. (2014) Hyponatremia: Pathophysiology, Classification, Manifestations and Management. International Urology and Nephrology, 46, 2153-2165. https://doi.org/10.1007/s11255-014-0839-2

[12] Sturdik, I., Adamcova, M., Kollerova, J., Koller, T., Zelinkova, Z. and Payer, J. (2014) Hyponatraemia Is an Independent Predictor of In-Hospital Mortality. European Journal of Internal Medicine, 25, 379-382. https://doi.org/10.1016/j.ejim.2014.02.002

[13] Madhuvan, H.S., Thimma Reddy, S.R., Ravishankar, S.N., Sadanand C.D. and Veerabhadra, G.K. (2019) A Prospective Study on Hyponatremia in Intensive Care Unit (ICU) Patients. IOSR Journal of Dental and Medical Sciences (IOSR-JDMS), 18, 17-20.

[14] Cecconi, M., Hochrieser, H., Chew, M., Grocott, M., Hoeft, A., Hoste, A., et al. (2016) Preoperative Abnormalities in Serum Sodium Concentrations Are Associated with Higher In-Hospital Mortality in Patients Undergoing Major Surgery. British Journal of Anaesthesia, 116, 63-69. https://doi.org/10.1093/bja/aev373

[15] Leung, A.A., Mcalister, F.A., Rogers Jr., S.O., Pazo, V., Wright, A. and Bates, D.W. (2012) Preoperative Hyponatremia and Perioperative Complications. Archives of Internal Medicine, 172, 1474-1481. https://doi.org/10.1001/archinternmed.2012.3992

[16] Peters, A.M., Seshadri, N., Neilly, M.D., Perry, L., Hooker, C.A., Howard, B., Sobnack, R., Irwin, A., Dave, S., Snelling, H., et al. (2013) Higher Extracellular Fluid Volume in Women Is Concealed by Scaling to Body Surface Area. Scandinavian Journal of Clinical and Laboratory Investigation, 73, 546-552. https://doi.org/10.3109/00365513.2013.819524

[17] Mannheimer, B., Skov, J., Falhammar, H., Calissendorff, J., Lindh, J.D. and Nathanson, D. (2019) Sex-Specific Risks of Death in Patients Hospitalized for Hyponatremia: A Population-Based Study. Endocrine, 66, 660-665.

https://doi.org/10.1007/s12020-019-02073-X

[18] Kayar, N.B., Kayar, Y., Ekinci, I., Erdem, E.D., Ismailova, M. and Sit, D. (2016) Relation between Severity of Hyponatremia and Comorbidity in Elderly Patients Who Develop Hyponatremia. Biomedical Research, 27, 872-876.

[19] Stachenfeld, N.S. (2014) Hormonal Changes during Menopause and the Impact on Fluid Regulation. Reproductive Sciences, 21, 555-561. https://doi.org/10.1177/1933719113518992

[20] Kirby, D.D. and Ames, D. (2001) Hyponatreamia and Selective Serotonin Re-Uptake Inhibitors in Elderly Patients. International Journal of Geriatric Psychiatry, 16, 484-493. https://doi.org/10.1002/gps.367

[21] Miller, K.C., Montain, S.J., Rehrer, N.J., et al. (2015) Statement of the 3rd Interna- 
tional Exercise-Associated Hyponatremia Consensus Development Conference, Carlsbad, California, 2015. British Journal of Sports Medicine, 49, 1432-1446. https://doi.org/10.1136/bjsports-2015-095004

[22] Peters, A.M., Glass, D.M. and Bird, N.J. (2011) Extracellular Fluid Volume and Glomerular Filtration Rate: Their Relation and Variabilities in Patients with Renal Disease and Healthy Individuals. Nuclear Medicine Communications, 32, 649-653. https://doi.org/10.1097/MNM.0b013e3283457466

[23] Saepudin, S., Ball, P.A. and Morrissey, H. (2015) Hyponatremia during Hospitalization and In-Hospital Mortality in Patients Hospitalized From Heart Failure. BMC Cardiovascular Disorders, 15, Article No. 88. https://doi.org/10.1186/s12872-015-0082-5

[24] Holmes, C.L., Landry, D.W. and Granton, J.T. (2003) Science Review: Vasopressin and the Cardiovascular System Part 2-Clinical Physiology. Critical Care, 8, Article No. 15.

[25] Pandey, V., Dutt, H.K., Singh, G., Amritha, P. and Vinod, A.P. (2017) Correlation of Blood $\mathrm{Na}^{+}$and $\mathrm{K}^{+}$Levels with Body Mass Index in Population of Garhwal Region of Uttarakhand. Journal of Applied Pharmaceutical Science, 7, 189-192. https://doi.org/10.7324/JAPS.2017.70127

[26] Qureshi, M.O., Khokhar, N., Saleem, A. and Niazi, T.K. (2014) Correlation of Hyponatremia with Hepatic Encephalopathy and Severity of Liver Disease. Journal of College of Physicians and Surgeons Pakistan, 24, 135-137.

[27] Verbalis, J.G., Barsony, J., Sugimura, Y., Tian, Y., Adams, D.J., Carter, E.A. and Resnick, H.E. (2010) Hyponatremia-Induced Osteoporosis. Journal of Bone and Mineral Research, 25, 554-563. https://doi.org/10.1359/jbmr.090827

[28] Drake-Holland, A.J. and Noble, M.I.M. (2016) The Hyponatremia Epidemic: A Frontiertoo Far? Frontiers in Cardiovascular Medicine, 3, 35. https://doi.org/10.3389/fcvm.2016.00035

[29] Israel, A. and Crossman, E. (2017) Elevated High-Density Lipoprotein Cholesterol Is Associated with Hyponatremia in Hypertensive Patients. The American Journal of Medicine, 130, 1324.E7-1324.E13. https://doi.org/10.1016/j.amjmed.2017.05.030

[30] Filippone, E.J., Ruzieh, M. and Foy, A. (2020) Thiazide-Associated Hyponatremia: Clinical Manifestations and Pathophysiology. American Journal of Kidney Diseases, 75, 256-264. https://doi.org/10.1053/j.ajkd.2019.07.011

[31] Drake-Holland, A.J. and Noble, M.I.M. (2011) Should We Now Abandon the LowSalt Diet? QJM, 104, 1013-1110. https://doi.org/10.1093/qjmed/hcr124

[32] Sahay, M. and Sahay, R. (2014) Hyponatremia: A Practical Approach. Indian Journal of Endocrinology and Metabolism, 18, 760-774. https://doi.org/10.4103/2230-8210.141320

[33] Jhaveri, K.D., Chawla, A., Xu, C. and Hazzan, A. (2014) Intravenous Albumin Infusion Is an Effective Therapy for Hyponatremia in Patient with Malignant Ascites. Indian Journal of Nephrology, 24, 51-53. https://doi.org/10.4103/0971-4065.125116

[34] Yamada, T., Morita, T., Furukawa, Y., Tamaki, S., Iwasaki, Y., Kawasaki, M., et al. (2018) Hyponatremia and Anemia Provide the Additional Long-Term Prognostic Information to ADHERE Risk Level in Patients Admitted for Acute Decompensated Heart Failure with Reduced or Preserved Left Ventricular Ejection Fraction. Circulation, 134, A14125. https://doi.org/10.1093/eurheartj/ehx504.P3386

[35] Tseng, C.K., Lin, C.H., Hsu, H.S., Ho, C.T., Huang, H.Y., Liu, C.S., et al. (2012) In Addition to Malnutrition and Renal Function Impairment, Anemia Is Associated with Hyponatremia in the Elderly. Archives of Gerontology and Geriatrics, 55, 77-81. 
https://doi.org/10.1016/j.archger.2011.06.019

[36] Lim, L.M., Tsai, N.C., Lin, M.Y., Hwang, D.Y., Lin, Y.H., Lee, J.J., Hwang, D.Y., Hung, C.-C. and Chen, H.-C. (2016) Hyponatremia Is Associated with Fluid Imbalance and Adverse Renal Outcome in Chronic Kidney Disease Patients Treated with Diuretics. Scientific Reports, 6, Article No. 36817.

https://doi.org/10.1038/srep36817

[37] Bhuvaneshwari, S., Saroj, P.V.S., Vijaya, D., Sowmya, S.M. and Kumar, S.R. (2018) Hyponatremia Induced by Angiotensin Converting Enzyme Inhibitors and Angiotensin Receptor Blockers-A Pilot Study. Journal of Clinical and Diagnostic Research, 12, FC01-FC03.

[38] Abdulla, M.C., Alungal, J. and Narayan, R. (2016) Recurrent Hyperkalemic Renal Tubular Acidosis and Hyponatremia in Diabetes Mellitus Due to Aldosterone Resistance. Journal of Integrative Nephrology and Andrology, 3, 29-30. https://doi.org/10.4103/2394-2916.175403

[39] Scott, J.H., Menouar, M.A. and Dunn, R.J. (2020) Physiology, Aldosterone Statpearls. Statpearls Publishing, Treasure Island, FL.

[40] Berardi, R., Caramanti, M., Castagnani, M., Guglielmi, S., Marcucci, F., Savini, A., et al. (2015) Hyponatremia Is a Predictor of Hospital Length and Cost of Stay and Outcome in Cancer Patients. Support Care Cancer, 23, 3095-3101. https://doi.org/10.1007/s00520-015-2683-Z

[41] Al Mawed, S., Shane Pankratz, V., Chong, K., Sandoval, M., Roumelioti, M.-E. and Unruh, M. (2018) Low Serum Sodium Levels at Hospital Admission: Outcomes among 2.3 Million Hospitalized Patients. PLoS ONE, 13, e0194379.

https://doi.org/10.1371/journal.pone.0194379

[42] Sato, N., Gheorghiade, M., Kajimoto, K., Munakata, R., Minami, Y., Mizuno, M., et al. (2013) Hyponatremia and In-Hospital Mortality in Patients Admitted for Heart Failure (From the ATTEND Registry). American Journal of Cardiology, 111, 10191025. https://doi.org/10.1016/j.amjcard.2012.12.019

[43] Reijnders, T.D.Y., Janssen, W.M.T., Niamut, S.M.L. and Kramer, A.B. (2020) Role of Risk Factors in Developing Osmotic Demyelination Syndrome during Correction of Hyponatremia: A Case Study. Cureus, 12, e6547.

https://doi.org/10.7759/cureus.6547 